\title{
Cutaneous Multispectral Optical tomography With ImageJ
}

\section{Tomografía Óptica Multiespectral Cutánea con ImageJ}

\author{
A. Aarón Muñoz Morales ${ }^{1,2 *}$, B. Sandra Vivas Toro ${ }^{1,3}$ \\ 1. Centro de Investigaciones Médicas y Biotecnológicas de la Universidad de Carabobo, Venezuela \\ 2. Departamento de Física, Facultad Experimental de Ciencias y Tecnología, Universidad de Carabobo, \\ Valencia, Venezuela \\ 2. Servicio de Dermatología, Ciudad Hospitalaria Dr. Enrique Tejera, Ciudad de Valencia, Carabobo, \\ Venezuela \\ ${ }^{(*)}$ E-mail: aamunozmorales@gmail.com
}

Received: 06/03/2016 Accepted: 03/12/2016

DOI: $10.7149 /$ OPA.49.4.49003

\begin{abstract}
:
The study of the optical properties of the skin has lead to the identification of the main chromophores such as hemoglobin and melanin. Melanin is a chromophore of the skin, whose accumulation in its different layers defines the types of melanocytic lesions (nevus). In order to achieve the reconstruction of acquired melanocytic lesions, the optic tomography of multispectral skin images was constructed by using a plugin created under the Image environment. This plugin demonstrates the iconographic overlap of images, by capturing segmented images (by multispectral bands), called the multispectral cube of the skin. This 3D image allowed to verify the location of the main chromophores of each layer by its relation to the histopathological location. With this method, the melanin pigment localization patterns (melanin nests) were defined in real time and the structural alterations of nevic skin lesions, that would otherwise not be visible without biopsy, could be defined. The method we present has been shown very useful for for locating the chromophores of the skin.
\end{abstract}

Key words: Multispectral, Skin, Photodiagnostic

\section{RESUMEN:}

Al estudiar las propiedades ópticas de la piel, se han identificado los principales cromóforos como son: la hemoglobina y la melanina. La melanina es un cromóforo de la piel, cuya acumulación en sus diferentes capas define los tipos de lesiones melanocíticas (nevus). Con la finalidad de lograr la reconstrucción de imágenes de lesiones melanocíticas adquiridas, se construyó la tomografía óptica de imágenes multiespectrales de piel mediante un plugin creado bajo el entorno ImageJ, que demuestra el solapamiento iconográfico de imágenes, mediante captura de imágenes segmentadas (por bandas multiespectrales), llamado cubo multiespectral de la piel. Esta imagen 3D permitió verificar la localización de los principales cromóforos de cada capa de la misma, al relacionarlas con la localización histopatológica. Con este método se definieron los patrones de ubicación de pigmento melánico (tecas de melanina), en tiempo real y se pudieron definir las alteraciones estructurales de lesiones névicas de piel que de otra forma no serían visibles, sin la toma de la biopsia. Este método se ha demostrado de gran utilidad para la localización de los cromóforos de la piel.

Palabras clave: Multiespectral, Piel, Fotodiagnóstico

\section{REFERENCES AND LINKS / REFERENCIAS Y ENLACES}

[1] Yamaguchi M, Mitsui M, Murakami Y, Fukuda H, Ohyama N, Kubota Y. "Multiespectral color imaging for dermatology: application in inflammatory and immunologic diseases." 13th Color Imaging Conference Final Program and Proceedings 7, 52-58 (2005) http://guchi.gsic.titech.ac.jp/NV/CIC13-Dermat-Proc.pdf.

[2] Vivas S, Pardo E, Martinez C, Alvarez I, Muñoz A. "Método Diagnóstico no Invasivos en Dermatología: Espectrofotometría de Reflexión Difusa," Informe Médico 17, 49-54 (2015). 
[3] Matts P.J, Dykes P.J and Marks R. “The Distribution of melanin in skin determined in vivo," British Association of Dermatologists 156, 620-628 (2007)

[4] Nishidate I, Maeda T, Niizeki K and Aizu Y, "Estimation of melanin and Hemoglobin Using Spectral Reflectance Images Reconstructed from a Digital RGB image by the Wierner Estimation Method," Sensor 13, 7902-7915 (2013) http://www.ncbi.nlm.nih.gov/pubmed/17493065

[5] Guolan L, Baowei F. "Medical Hyperspectral imaging: a review," J. Biomed Opt. 19, 10901 (2014) https://doi.org/10.1117/1.JB0.19.1.010901

[6] Kardynal A, Olszewska M. "Modern non-invasive diagnostic techniques in the detection of early cutaneous melanoma," J. Dermatol. Case. Rep 8, 1-8 (2014) https://doi.org/10.3315/idcr.2014.1161

[7] Wang F, Behrooz A, Morris M, Adibi A. "High-Contrast Subcutaneous Vein Detection and Localization Using Multispectral Imaging," J. Biomed. Opt. 18, 1-3 (2013) https://doi.org/10.1117/1.JB0.18.5.050504

[8] Alex A, Povazay B, Hofer B, Popov S, Glittenberg C, Binder S, Drexler W. "Multiespectral in vivo threedimensional optical coherence tomography of human skin,” J. Biomed. Opt. 15 (2010). https://doi.org/10.1117/1.3400665

[9] Jolivot R, Benezeth Y, Marzani F. "Skin Parameter Map Retrieval from a Dedicated Multispectral Imaging System Applied to Dermatology/Cosmetology,". International Journal of Biomedical Imaging; 2013, 978289 (2013) https://doi.org/10.1155/2013/978289

[10] Schneider, C.A., Rasband, W.S., Eliceiri, K.W. "NIH Image to ImageJ: 25 years of image analysis," Nature Methods 9, 671-675, (2012)

https://doi.org/10.1038/nmeth.2089

\section{Introducción}

El análisis de la interacción de la luz con la piel humana es una alternativa para el diagnóstico de tejidos anómalos estableciéndose en función de ello, técnicas no invasivas que permiten diagnosticar las lesiones cutáneas en sus diferentes estados (desde su fase inicial) [1-4]. Una de estas técnicas es el estudio de las Imágenes Multiespectrales.

El estudio de las Imágenes Multiespectrales se basa en los diferentes fenómenos ópticos (principalmente absorción y el esparcimiento) que ocurren cuando la luz incide sobre las diferentes capas de la piel (Epidermis, Dermis, Hipodermis) para luego emerger del interior de la piel como luz reflejada de forma difusa. Esto permite la visualización de las estructuras internas, proveyendo imágenes secuenciales en las diferentes bandas espectrales, dando origen a la Dermatoscopia Multiespectral [5, 6].

En el presente trabajo de investigación se hace una reconstrucción de imágenes de lesiones melanocíticas adquiridas mediante el desarrollo de un plugin bajo el entorno Imagej. Éste realiza un solapamiento iconográfico, para las diferentes bandas espectrales a fin de obtener una Tomografía Óptica Axial y Transversal de la piel humana con el fin de analizar la distribución de los cromósforos en las diversas capas.

\section{Materiales y métodos}

En primer lugar se llevó a cabo la obtención de las imágenes, empleando un Microscopio Epiluminiscente con aumento máximo de 200X, a fin de visualizar las estructuras no perceptibles a simple vista. Esta técnica ampliamente usada en la Dermatología Tradicional (Dermatoscopia clásica), permite evidenciar características morfológicas en su superficie de las lesiones pigmentadas que mejoran la aproximación diagnóstica. [6] Sin embargo, la técnica de Microscopía Epiluminiscente Clásica, resulta insuficiente como método, en la obtención de información sobre las estructuras internas y la profundidad. [3]

Con el fin de mejorar la técnica se desarrolló un dispositivo que permitiera obtener un grupo de imágenes multiespectrales en el rango del visible. Se emplearon ocho LEDs (Light-Emitting Diode) tricromáticos de alto brillo que iluminan a la superficie cutánea con seis 6 colores de forma independientes (Figura 1).

A través de esta técnica se aumenta el contraste en las imágenes para cada banda, lo cual permite la observación de la distribución de los cromóforos que componen a la lesión [7, 8]. Cabe destacar, que se debe considerar el tiempo de exposición del sensor de la cámara para cada color, con el fin de lograr intensidades de respuesta similares. Es de importancia hacer notar, que en el proceso de construcción de 
la imagen en tres dimensiones, se debe tomar en cuenta la radiancia de cada banda ya que ellopermite conocer la profundidad de la lesión.
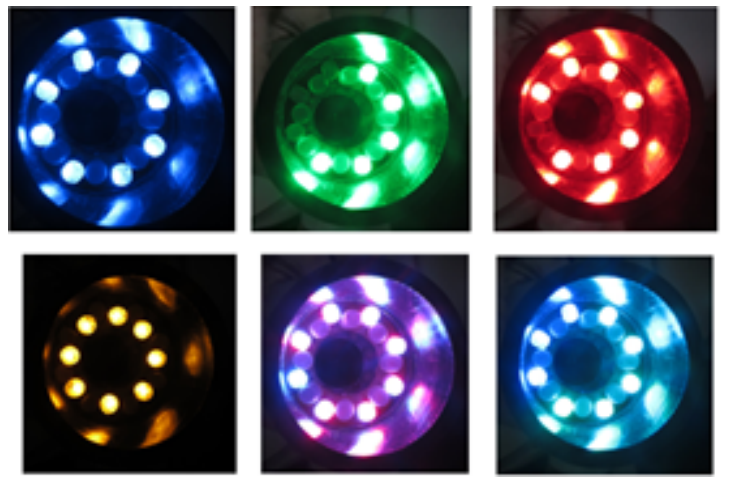

Fig. 1. Sistema de LEDs tricromático de alto brillo desarrollado para obtención de las imágenes multiespectrales (Azul, Verde, Rojo, Amarillo, Magenta y Cian).

En la piel la absorción está determinada por la presencia de Melanina y Hemoglobina, [9] siendo estas las que determinan la profundidad de penetración de la luz (Figura 2). Al seleccionar la banda adecuada que contenga las longitudes de onda próximas al máximo del espectro de absorción de estos pigmentos, se mejora el contraste de la imagen de la lesión [7].

Considerando todo lo anterior, se desarrolló un plugin en código Java llamado "Analisis_Multiespectral”, para ser implementado en el programa de procesamiento de imágenes ImageJ (Image Processing and Analysis in Java) [10]. La finalidad del plugin es lograr el solapamiento de las imágenes para la obtención de un cubo multiespectral, el cual representa la Tomografía Óptica con resolución Axial (TOA) y Transversal (TOT) de la piel que definiremos como “Tomografía Óptica Multiespectral Cutánea” (Figura 3).

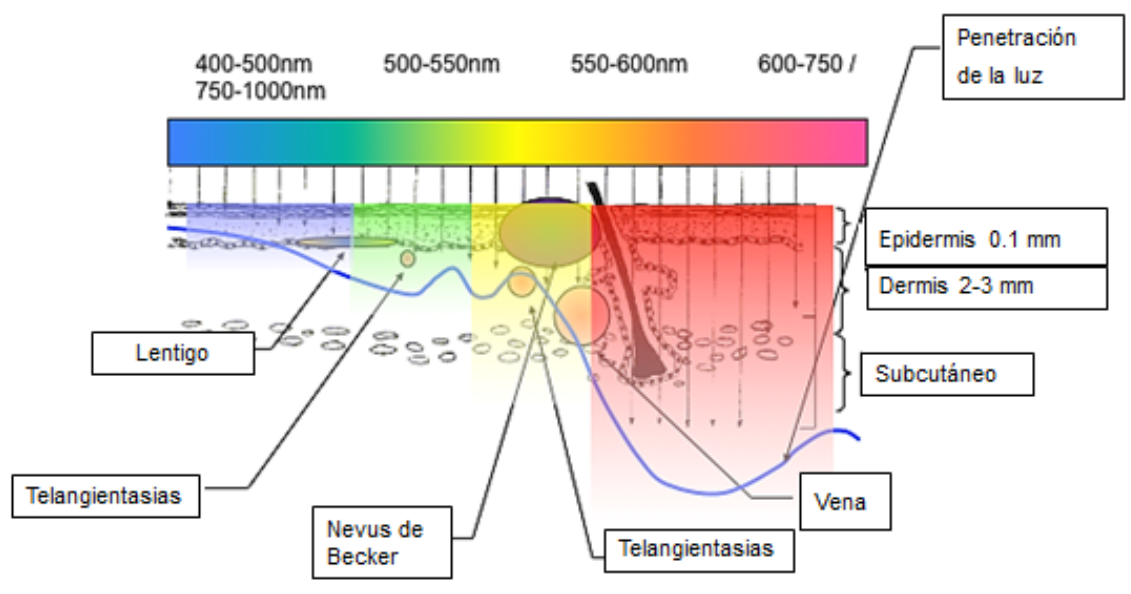

Fig 2. Penetración de las diferentes longitudes de onda en la piel [7] 


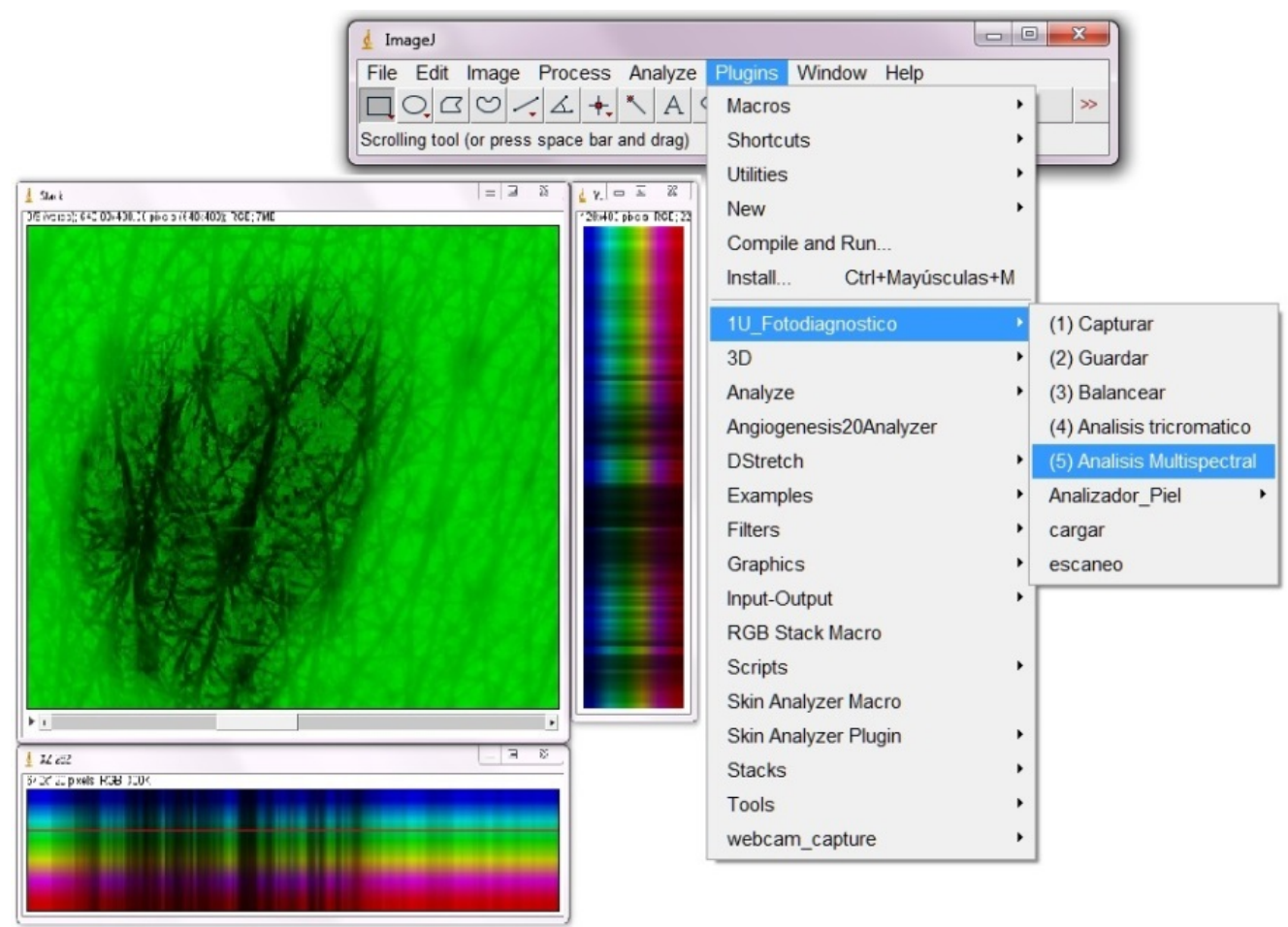

Fig. 3. Tomografía Óptica con resolución Axial (TOA) y Transversal (TOT) de la piel.

\section{Resultados obtenidos}

Para la verificación de las Tomografías Ópticas Multiespectrales Cutáneas desarrolladas, se realizó un análisis de lesiones melanocíticas adquiridas. Para ello se seleccionaron tres lunares calificados como Nevus de Unión, Nevus Intradérmico y Nevus Displásico (clasificación histopatológica que se hace en función de la ubicación de las tecas de melaninas en las capas de la piel: Epidermis, Intradermis y Dermis respectivamente). Los diagnósticos fueron realizados a través de la Biopsia Óptica y bajo Consentimiento Informado de los pacientes que asistieron al servicio de dermatología de la Ciudad Hospitalaria Dr. Enrique Tejera, de la Ciudad de Valencia, Venezuela, siguiendo los códigos de Bioética establecidos [8].

En el primer caso se tiene un Nevus de Unión, caracterizado por ser un tipo de lunar superficial con concentraciones de tecas melánicas en la capa más superficial de la piel (como es la epidermis). Se puede observar una mayor concentración de puntos negros (tonalidades oscuras) en la banda No.1 con un rango de longitudes de onda entre 400nm y 450nm (ver figuras 4 y 5), obteniéndose información de la Melanina dispuesta en la Epidermis y estimar la distribución de los cromósforos en las capas de la piel.

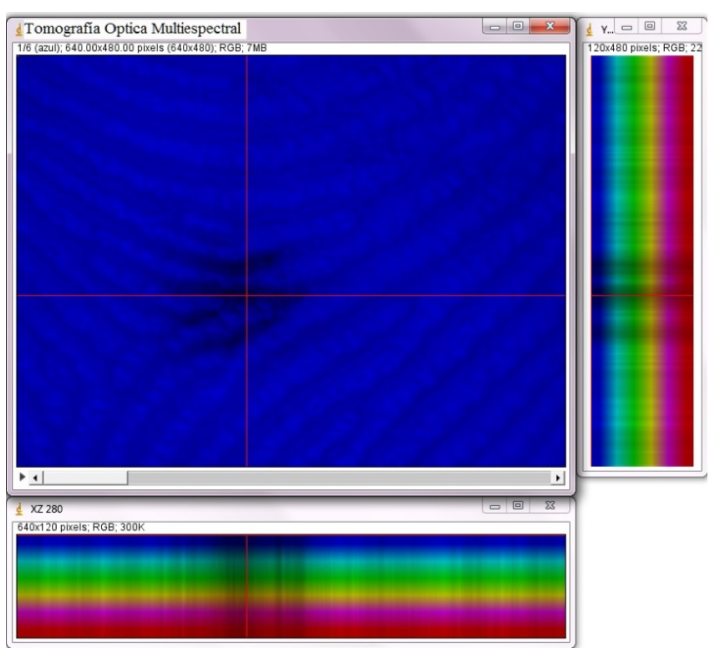

Fig. 4. Tomografía Axial y Transversal Cutánea. 
Para el segundo caso se presenta un Nevus Intradérmico (figura 6), caracterizado por ser un tipo de lunar donde las concentraciones de las tecas melánicas se encuentran en la unión Intradermis-Dermis de la piel. Se puede observar en la tomografía una mayor concentración de puntos negros (tonalidades oscuras) en la banda No. 3 correspondiente al rango espectral entre $500 \mathrm{~nm}$ y $550 \mathrm{~nm}$, logrando una mayor nitidez de la imagen en el color verde.
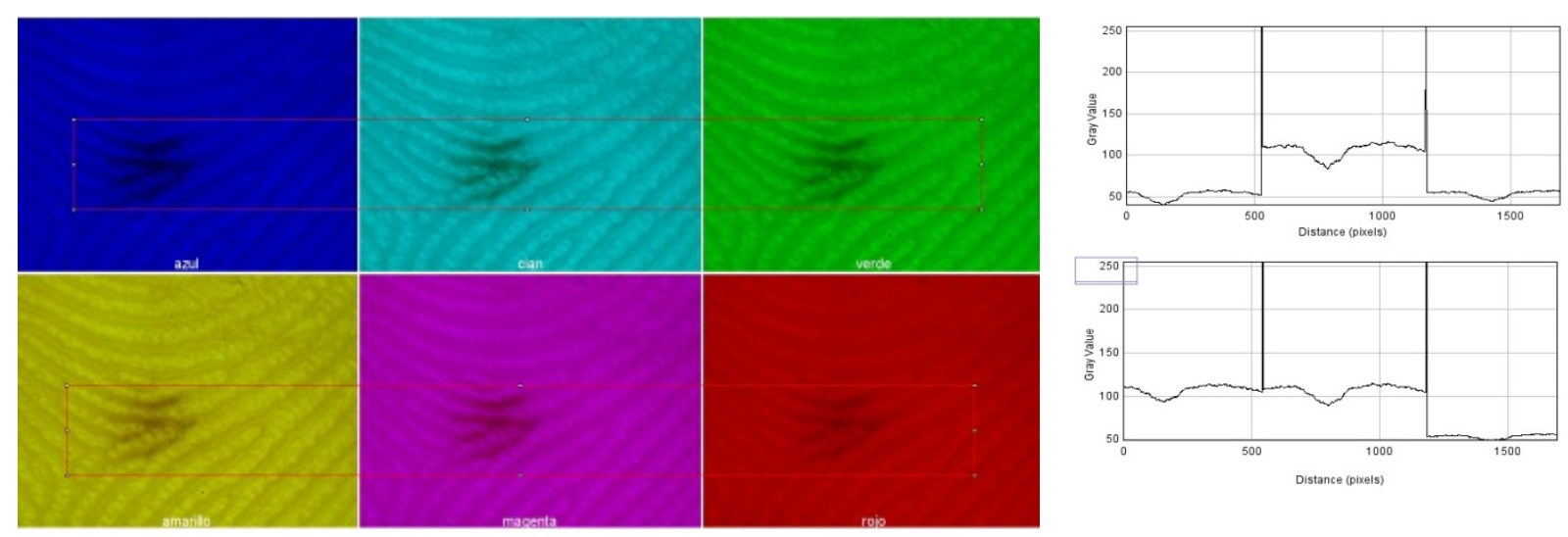

Fig. 5. Distribución de los centros de absorción por cada segmento de la tomografía
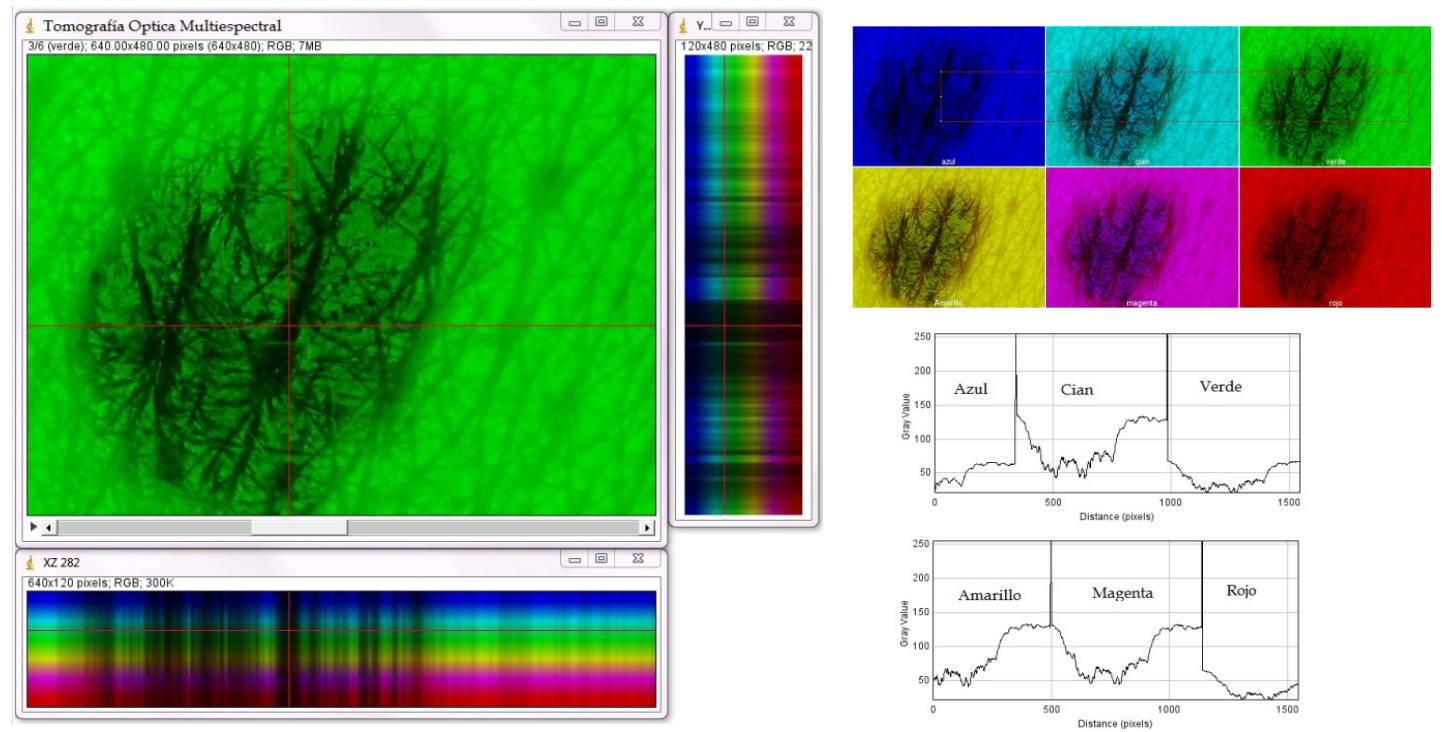

Fig. 6. Tomografía Óptica Multiespectral de un Nevus de Intradérmico.

Por último se presenta un Nevus Displásico (Fig. 7), caracterizado por ser un tipo de lesión melanocítica más profunda al ubicarse en la Dermis (es importante señalar, que es el más preocupante para los especialista en dermatología, por ser el precursor del melanoma), observándose fácilmente que la mayor absorción se encuentra en la banda No. 6, correspondiente a las longitudes de onda entre $650 \mathrm{~nm}-700$ $\mathrm{nm}$, lo cual permite afirmar y confirmar que la lesión efectivamente está presente en la Dermis. 


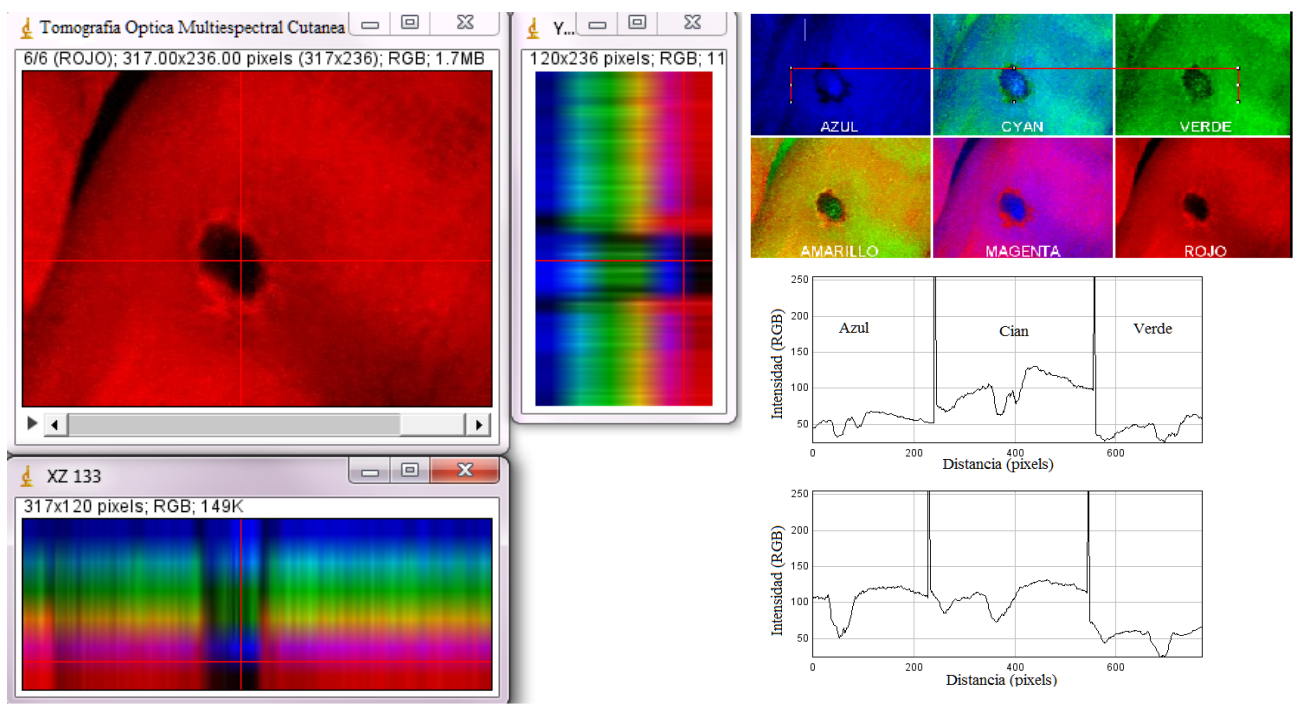

Fig .7. Tomografía Óptica Multiespectral de Nevus Displásico

\section{Conclusión}

Finalmente se concluye que el desarrollo e innovación de la Técnica de Tomografía Óptica Multiespectral Cutánea, se presenta como una verdadera herramienta óptica no invasiva para la monitorización de las lesiones pigmentada en la piel humana, afín de co-ayudar en la precisión diagnóstica en el área de Dermatología, puesto que permite, la evaluación de lesiones melanocíticas adquiridas y su potencialidad en el diagnóstico precoz del melanoma. 